\title{
Sonic Rhinoplasty: Review and Updated Uses
}

\author{
Nikolaus Hjelm, MD ${ }^{1}$ Jared Goldfarb, MD ${ }^{1} \quad$ Howard Krein, MD, $\mathrm{PhD}^{1} \quad$ Ryan N. Heffelfinger, MD ${ }^{1}$ \\ Edmund Pribitkin, MD, MBA, FACS ${ }^{1}$
${ }^{1}$ Department of Otolaryngology-Head and Neck Surgery, Thomas Jefferson University Hospital, Philadelphia, Pennsylvania
Facial Plast Surg 2021;37:107-109.

\begin{abstract}
Address for correspondence Nikolaus Hjelm, MD, Department of Otolaryngology-Head and Neck Surgery, Thomas Jefferson University Hospital, 925 Chestnut Street, 6th Floor, Philadelphia, PA 19107 (e-mail: Nikolaus.hjelm@jefferson.edu).
\end{abstract}

\author{
Abstract \\ Keywords \\ - sonic rhinoplasty \\ - ultrasonic bone \\ aspirator \\ - functional rhinoplasty \\ - cosmetic rhinoplasty
}

The ultrasonic bone aspirator (UBA) allows for precision and provides ease of use in multiple steps of cosmetic and functional rhinoplasties. Its use has previously been described; however, since the publication of many studies the UBA has been improved and its applications for rhinoplasty have been modified and expanded. Both bony and cartilaginous modifications to the nose can be aided with the use of the UBA. From our experience, patient results have subjectively been found to be more acceptable, if not better, than with the use of other rhinoplasty instruments.

The use of the ultrasonic bone aspirator (UBA) for rhinoplasty has been previously described. ${ }^{1-4}$ However, since the publication of these articles, the UBA has been improved and its uses for rhinoplasty have been modified and expanded.

The specific UBA device used at our institution is the Sonopet (Stryker Neuro Spine ENT). The Sonopet, as described by its manufacturer, emulsifies bone with ultrasonic vibration technology. The operative handpiece tip couples longitudinal vibration with torsional oscillation. This technology allows for smooth bone cuts while effectively minimizing trauma to surrounding and underlying soft tissue. The degree of bone removal is dependent on the geometry of the surgical tip, the power setting of the console, the density of the bone, and the amount of time the surgical tip spends in contact with the bone. In general, the greater the power setting and the less dense the bone, the faster and easier the handpiece tip cuts through the bone. The Sonopet is able to move soft tissue away from the vibrating and oscillating tip, while bone is unable to. ${ }^{5}$ We have found the bone tips to be effective not only for bone cuts but in the contouring of cartilage as well.

Original descriptions of Sonopet use for rhinoplasty have discussed its application for multiple aspects of cosmetic and functional rhinoplasty. ${ }^{1-4}$ We describe our experience and techniques gained over the past 5 years of using the Sonopet for open approach rhinoplasty.

\section{Uses}

\section{Dorsal Hump Reduction with Concurrent Glabellar Reduction}

Once a soft-tissue envelope is elevated and osteotomies have been performed, our attention turns to the bony and cartilagi-

published online August 24, 2020
Issue Theme Preservation Rhinoplasty: An Update; Guest Editor: Jose Carlos Neves, MD nous dorsal hump. Our preference is to use an Aufricht retractor to elevate the soft-tissue envelope to allow full visualization. ${ }^{1,3}$ We proceed to lower the cartilaginous septum with a scalpel and removed the excised septal cartilage from the field. We previously used the Sonopet's "Spetzler Claw" handpiece tip to smooth and reduce any remaining cartilaginous or bony irregularities. However, since the development of Sonopet's newer "Payner 360" handpiece tip, we now address the dorsal hump with this modification (-Video $\mathbf{1}$ ). We have found that this 360-degree tip provides an easier ability to precisely contour the bony and cartilaginous aspects of the dorsal hump due to its ability to move the handpiece side to side. The "Spetzler Claw" only allows for a front-to-back motion.

\section{Video 1}

Demonstrations of Sonopet use with the "Payner 360" and "Spetzler Claw" handpiece tips. Videos were obtained from the senior authors (E.P., R.N.H., H.K.) during several different rhinoplasties; use is tailored to the needs of the patient and goals of the operation. (Presented with permission of Jefferson Facial Plastic Surgery). Online content is viewable at: https://www. thieme-connect.com/products/ejournals/html/ 10.1055/s-0040-1714673.

Similar to the side-to-side technique used with a diamond burr drill, the Payner tip allows us to contour and reduce the glabella in a controlled and precise fashion. As compared (c) 2020. Thieme. All rights reserved. Thieme Medical Publishers, Inc., 333 Seventh Avenue, 18th Floor, New York, NY 10001, USA
DOI https://doi.org/ 10.1055/s-0040-1714673. ISSN 0736-6825. 
with a drill, the Sonopet does not skip off of bone which would put the surrounding soft tissue at risk of injury. ${ }^{1,3}$ Our observed results between handpiece tips are similar for glabellar and hump reduction, but we believe contouring in a side-to-side motion with the "Payner 360" reduces operative time and allows for easier use.

\section{Sculpting of Mobile Fragments and Recontouring Nasal Bones}

Similar to our approach for the dorsal hump, we have adopted the "Payner 360" tip over the "Spetzler Claw" for contouring of the mobile nasal bones following osteotomies. Visualization of deformities is provided with an Aufricht retractor to elevate the soft-tissue envelope off of the fractured nasal bones. Using the Sonopet to smooth these irregularities is not only important for thin skinned patients but also can reduce trauma in all patients when the alternative rasp or drill is used. ${ }^{1}$ Although we do not use the device for osteotomies, Cochran and Roostaeian have found success when using the UBA for this procedure. ${ }^{6}$

\section{Septoplasty}

The Sonopet can be used to precisely address potentially problematic deviations of the nasal septum in a controlled, targeted fashion. Although complete mucoperichondrial flap elevation is often necessary for large deviations or required for cartilage harvest, in instances of isolated septal spurs a small incision can be made at the anterior leading edge of a deviation. ${ }^{1}$ The overlying cartilage and bony spur are then reduced with the "Payner 360" tip. This technique leaves the contralateral mucoperichondrial flap undisturbed, thus reducing the risk of perforation or hematoma along with minimizing the need for nasal packing or splinting.

The Sonopet can also be used for the controlled reduction of a widened or deviated maxillary crest ( - Video 1). Using this technique is a controlled alternative to the use of osteotomes which may result in bony abnormalities, sharp projections, and perforations of the mucoperichondrial flap.

Additionally, the Sonopet may be used to safely address and reduce deviations of the superior bony septum. The controlled reduction of this region helps in preserving the keystone area and thus the stability of the dorsal septum. Its use also helps reduce risk of torque on the ethmoid plate and skull-base, which could potentially cause a cerebrospinal fluid leak. ${ }^{7}$

\section{Sculpting of Upper and Lower Lateral Cartilage, Septal and Rib Cartilage Grafts}

An undescribed use of the Sonopet is its ability to shape free autologous or allogeneic cartilage. Once septal or rib cartilage is harvested or allogeneic rib cartilage is brought to the field for nasal grafts, the Sonopet with the "Payner 360" tip is found to be useful for shaping the cartilage graft ( - Video 1 ). Traditionally, we have used a scalpel to shape and shave these cartilage grafts to a desired form. Although the scalpel has been effective at acquiring the desired size and shape of our grafts, we found the Sonopet to be effective for small and quick modifications. Using the "Payner 360" tip, cartilage graft edges are easy to contour as needed.
The lower and upper lateral cartilages may be modified with utilization of either the "Spetzler Claw" or "Payner 360" handpiece tip. Cartilage irregularities that may appear in a thin-skinned patient can be smoothed in a controlled manner with the use of the Sonopet (- Video 1 ). In addition to contouring, the Sonopet also allows for the surgeon to excise unwanted or excess cartilage.

\section{Piriform Aperture Enlargement}

First described in 2015 by Roy et al, the Sonopet can be used for piriform aperture enlargement in a functional rhinoplasty. ${ }^{8}$ The nasal valve is bounded laterally by the nasal process of the maxilla and head of the inferior turbinate concha. By incising through the mucosa at the height of the head of the inferior turbinate concha and elevating the mucosa and periosteum with a cottle, the head of the concha and lateral process of the maxilla is exposed. The Sonopet, affixed with the "Spetzler Claw," is then used in a back-and-forth motion to emulsify the desired amount of bone to widen the piriform aperture and expand the nasal airway. The non-serrated back side of the "Spetzler Claw" in this procedure protects the surrounding soft tissue that will inevitably lay against the Sonopet during its use. This prevents unnecessary trauma while effectively removing bone within a small surgical area. $^{8}$

\section{Inferior Turbinate Reduction}

Although numerous techniques for inferior turbinate reduction have been described and are widely used, we have found the ultrasound bone aspirator in a submucosal plane to be a safe, efficient, and effectual approach. ${ }^{1}$ Preparation for our reduction first begins with topically vasoconstricting the turbinate mucosa with oxymetazoline pledgets followed by injection with lidocaine with epinephrine. The turbinate is then fractured medially. We then sharply incise the mucosal head of the inferior turbinate down to bone. A cottle is then used to submucosally and freely dissect a plane overlying the bone of the inferior turbinate. The Sonopet using a "Spetzler Claw" tip is then introduced into this plane with the aspirating surface of the tip facing the conchal bone (-Video 1 ). The bone is then reduced systematically and uniformly along the entire length. Care and attention are given to ensure that the proximal end of the Sonopet does not make contact with the nasal ala, columella, or sill during this maneuver. Advantageously, the lack of trauma to the mucosa prevents future formation of synechia. ${ }^{1}$ The use of this technique has been previously demonstrated to be a safe and efficient technique in addressing inferior turbinate hypertrophy resulting in mean reduction of Nasal Obstruction Symptom Evaluation scale scores from 51 to $25 .^{4}$

\section{Reducing the Nasal Spine}

Traditionally, the reduction of the anterior nasal spine necessitates the use of rongeurs or osteotomes which can result in bony abnormalities and sharp projections. These bony irregularities are then typically addressed with rasping which can result in overresection of the spine due to the challenging and imprecise nature of the technique. ${ }^{1}$ 
Overresection of the anterior nasal spine can lead to cosmetic and functional consequences in the form of reduced nasal tip support. ${ }^{9}$ We found the use of the "Payner 360" tip to be efficient in precisely reducing and smoothly contouring the anterior nasal spine, limiting chances of over resection (-Video 1).

\section{Discussion}

All the authors of this article are facial plastic and reconstructive surgeons, among which three are senior authors (E.P., H.K., R.N.H.). They found subjective success with the use of the Sonopet, each using the device to different degrees due to personal preference. The instrument has proven to be an effective tool for all of their cosmetic and functional rhinoplasty techniques.

Sonopet currently has two console models, three different handpieces, and multiple soft tissue and bone-specific handpiece tips. ${ }^{5}$ We have consistently used the original "Sonopet Console" and "Universal 25-kHz Angled Handpiece." Previous publications of Sonopet use have only described use of the 2.8-mm-width "Spetzler Claw" handpiece tip; however, we have adopted the use of the 3.12-mm-width "Payner 360" handpiece tip and have noted improved functionality in some of our operative techniques. ${ }^{1-4}$ We have neither used the latest model of console "Sonopet iQ" nor the latest model of handpiece "34-kHz Angled Handpiece." Both allows for faster cutting and more precision. ${ }^{5}$ The Sonopet is primarily marketed and has been studied to be effective for orthopaedic and neurosurgical use, but facial plastic and reconstructive surgery is also listed as an application. ${ }^{10,11}$ The "Piezoelectric System" is another ultrasonic surgical device often used by facial plastic surgeons. ${ }^{12-14}$ However, we do not have this device at our institution. Studies comparing the "Piezoelectric System" with the Sonopet would be beneficial to the surgical community.

According to Greywood et al, the Sonopet is an expensive equipment that may best serve surgeons who can split the cost within a surgical group or within a hospital setting. ${ }^{4}$ Other previously described uses of the device have been noted to include dacryocystorhinostomy, endoscopic transorbital decompression, and functional endoscopic sinus surgery. ${ }^{15-17}$ Due to the size of the handpiece, an image guidance apparatus can be affixed to it, allowing for safe endonasal use. The multifunctionality may make the device a more cost-effective purchase, but it is dependent on each surgeon's extent of use and comfortability with the instrument. No major complications have been experienced by the senior authors (E.P., H.K., R. N.H.). However, there is the risk of burns to the nasal vestibule if the Sonopet is not sheathed.

\section{Conclusion}

The UBA allows for precision and provides ease of use in multiple steps of cosmetic and functional rhinoplasties. The Sonopet's "Payner 360" tip has enabled even more efficiency and maneuverability as compared with the "Spetzler Claw."
Patient results have subjectively been found to be more acceptable, if not better, than with the use of other rhinoplasty instruments. Further studies are needed to determine if the device does improve operative time and, as a result, is cost-effective.

Note

No previously related publications of the same study.

Funding

None.

Conflict of Interest

None declared.

\section{References}

1 Pribitkin E, Greywoode JD. Sonic rhinoplasty: innovative applications. Facial Plast Surg 2013;29(02):127-132

2 Greywoode JD, Pribitkin EA. Sonic rhinoplasty: histologic correlates and technical refinements using the ultrasonic bone aspirator. Arch Facial Plast Surg 2011;13(05):316-321

3 Pribitkin EA, Lavasani LS, Shindle C, Greywoode JD. Sonic rhinoplasty: sculpting the nasal dorsum with the ultrasonic bone aspirator. Laryngoscope 2010;120(08):1504-1507

4 Greywoode JD, Van Abel K, Pribitkin EA. Ultrasonic bone aspirator turbinoplasty: a novel approach for management of inferior turbinate hypertrophy. Laryngoscope 2010;120(Suppl 4):S239

5 Stryker, "Sonopet." Stryker. Available at: https://www.stryker.com/us/en/nse/products/sonopet-ultrasonic-aspirator.html. Accessed

6 Cochran CS, Roostaeian J. Use of the ultrasonic bone aspirator for lateral osteotomies in rhinoplasty. Plast Reconstr Surg 2013;132 (06):1430-1433

7 Ketcham AS, Han JK. Complications and management of septoplasty. Otolaryngol Clin North Am 2010;43(04):897-904

8 Roy S, Iloreta AM, Bryant LM, Krein HD, Pribitkin EA, Heffelfinger RN. Piriform aperture enlargement for nasal obstruction. Laryngoscope 2015;125(11):2468-2471

9 Marianetti TM, Boccieri A, Pascali M. Reshaping of the anterior nasal spine: an important step in rhinoplasty. Plast Reconstr Surg Glob Open 2016;4(09):e1026

10 Inoue T, Ikezaki K, Sato Y. Ultrasonic surgical system (SONOPET) for microsurgical removal of brain tumors. Neurol Res 2000;22 (05):490-494

11 Hosseini P, Mundis GM Jr, Eastlack R, et al. Is there a role for an ultrasonic bone-cutting device in adult spinal deformity: a safety and reproducibility study. Shafa Ortho J 2016;3(03):e7461

12 Bertossi D, Albanese M, Mortellaro C, et al. Osteotomy in genioplasty by piezosurgery. J Craniofac Surg 2018;29(08):2156-2159

13 Bertossi D, Nocini R, Luciano U, et al. Piezoelectric surgery inserts vs conventional burst: a clinical investigation. J Biol Regul Homeost Agents 2018;32(02, Suppl 2):15-19

14 Bertossi D, Lucchese A, Albanese M, et al. Piezosurgery versus conventional osteotomy in orthognathic surgery: a paradigm shift in treatment. J Craniofac Surg 2013;24(05):1763-1766

15 Sivak-Callcott JA, Linberg JV, Patel S. Ultrasonic bone removal with the Sonopet Omni: a new instrument for orbital and lacrimal surgery. Arch Ophthalmol 2005;123(11):1595-1597

16 Chappell MC, Moe KS, Chang SH. Learning curve for use of the Sonopet ultrasonic aspirator in endoscopic dacryocystorhinostomy. Orbit 2014;33(04):270-275

17 Bruggers S, Sindwani R. Evolving trends in powered endoscopic sinus surgery. Otolaryngol Clin North Am 2009;42(05):789-798, viii 